\title{
Early Warning and Control Of Tourism Crisis
}

\author{
Zhaoting Tong \\ Institute of tourism , Zhenjiang college Zhenjiang Jiangsu 212003, China \\ paperiset@163.com
}

Keywords: Tourism crisis, network public opinion, monitoring and warning.

\begin{abstract}
To effectively deal with all kinds of tourism crisis of network public opinion, repair and maintenance the damaged image of the government, enterprises and individuals, the method of the network public opinion monitoring and warning is necessary. Using the ticket fee events of the ancient city phoenix's scenic spot for an example, we have made a research. It can be seen that the network public opinion of tourism crisis event's risk index has a certain indicative and predictability.
\end{abstract}

\section{Introduction}

Network public opinion of tourism crisis is the products of Internet users and media for tourism phenomenon and the crisis events related to the tourism phenomenon, include their expression of cognition, emotion, attitude, opinion and behavior etc.. Network public opinion of tourism crisis includes the topics like the pohlice and people livelihood, the government management, visits and foreign communication, social culture, tourist market etc., meanwhile it also involves the tourism system such as transportation, destination, the development environment etc..

Network public opinion monitoring and warning is one important ring of network public opinion management, which provides the opportunity for managers with identification network public opinion risk and crisis, evaluating the size of public opinion events harm and taking the corresponding effective measures. To effectively deal with all kinds of tourism crisis of network public opinion, repair and maintenance the damaged image of the government, enterprises and individuals, the method of the network public opinion monitoring and warning is necessary. The existing research on the dimension of the index system mainly centered on public opinion content [1], public opinion media, the events causing public opinion [2] and the net-citizens who receives information and fabricates perspective [3], etc. The method of determining the index weight includes the analytic hierarchy process, the BP neural network [4], gray prediction method [5], etc..

On the basis of the above research, from the composition and connotation of network public opinion of tourism crisis events, it designs index assessment system of the network public opinion of tourism crisis monitoring and warning and makes an empirical research in this paper, which provides the thinking method for the management of network public opinion of tourism crisis.

\section{The Selection of Index}

According to the purpose of the research, index can accurately reflect the situation of the study object. The establishment of the index system is the process of that the identifier of one aspect of the abstract research object is decomposed into the behavior, operational the structure according to its nature and characteristics.

According to the principle of comprehensive combing, extract 84 indicators as an alternative, informative data provided by experts, not anonymous, increase communication experts, delete don't agree on some way, such as to modify the Delphi Method (Delphi Method) (see figure 1). 


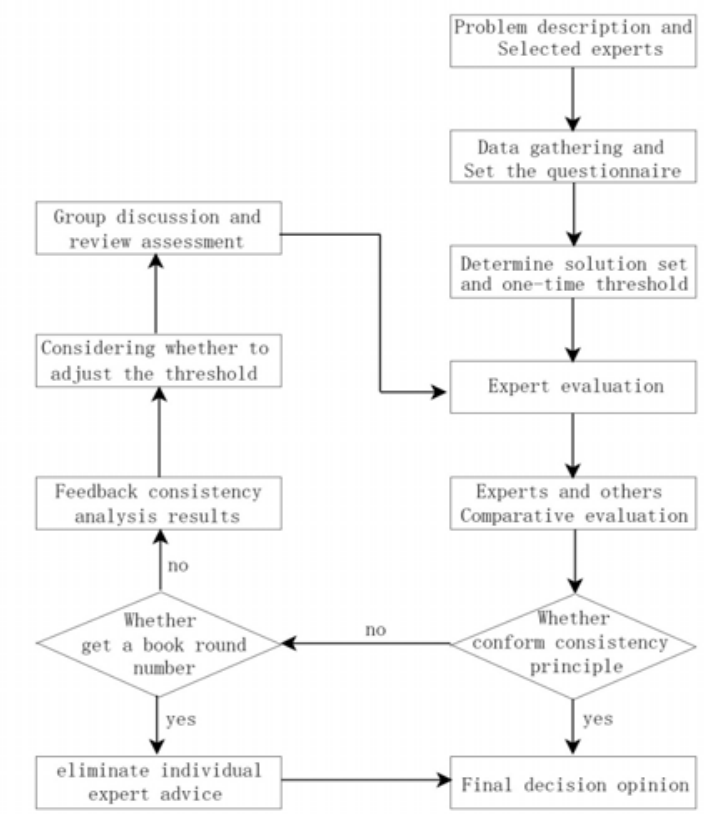

Fig. 1 The decision-making process of modified Delphi method

For the tourism safety and crisis management, the network of public opinion of the new media and network related experts and professors, graduate students and the personage inside course of study for the first time in a survey questionnaire for the importance of alternative indicators ( 5 point scale: 1 is not important, 2 say is not important, 3 said general said, 4, 5, said very important) and acquired (2 point scale: 1 easy to obtain, says it is not easy to get 2) to investigate, through distribution, mail questionnaire face to face, E-mail, twitter direct messages way questionnaire 20, recycling questionnaire 15 , recovery rate was $75 \%$, including effective 13 questionnaires, effective recovery rate was $65 \%$. Factor analysis with the result of questionnaire, calculate crowns Bach alpha reliability coefficient calculates reliability questionnaire results, calculation of quantity of Sampling Adequacy Test (Kaiser - Meyer - Olkin Measure of from Adequacy) and Bartlett sphere Test (Bartlett' s Test of Sphericity) to Measure the validity of results of the questionnaire survey. Object the subject of public opinion, public opinion and public opinion of three dimensions of ontology Cronbach 's alpha value respectively 0.732 and 0.744 and 0.705 , were 0.70 or above, point out that the reliability of the questionnaire survey results with high and internal stability, the reliability is good. Sampling adequacy measure test value is $65.476 \%$, good reliability and validity of. Based on the questionnaire survey results screening, complement, perfect, get index system (It's seen in figure 2).

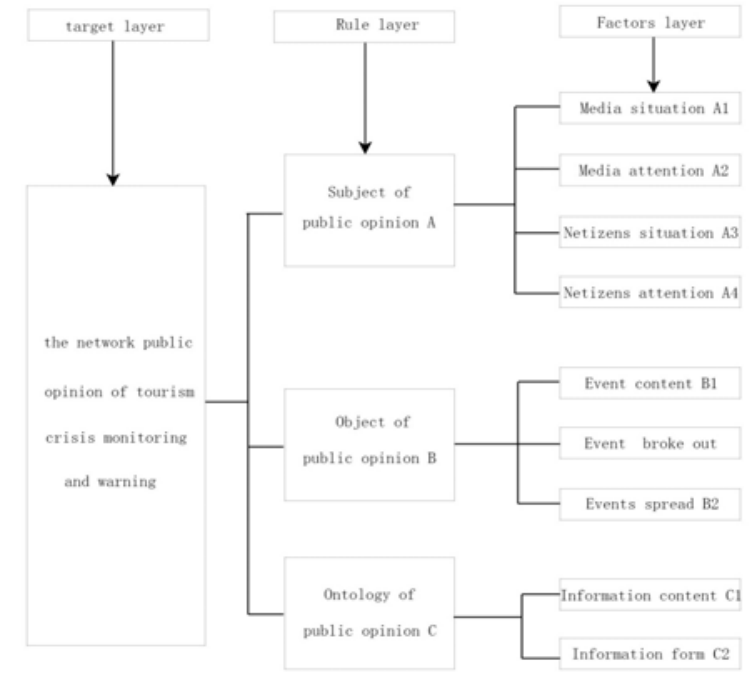

Fig. 2 The evaluation model tree of public opinion monitoring and warning 


\section{Quantization of Indicators}

The Measuring Method. The methods of access to the data at present, there are mainly the questionnaire survey, the actual observation, content analysis and statistics, etc.

Quantitative Methods. The quantization processing of the data obtained from the measurement mainly relay to refer to the statistics data, the direct description. And then the index is made for a quantization and to acquire the precise value.

Properties of Index. It includes the positive and negative indicators. While the value of the positive indicator is greater, the value of the index is higher, which is also known as performance indicators; While the value of the negative indicator is lesser, the value of the index is higher, which is also known as cost index.

The Standardization of the Index. By the poor standardization method, through compared with the reference (the maximum and the minimum of the risk), to make a standardization for the score. The risk index ${ }^{y_{11}}$ of the positive index is as follows:

$$
y_{i j}=\left(x_{i 1}-\operatorname{mlnx}_{i_{1}}\right) /\left(\operatorname{maxx}_{i_{1}}-\operatorname{mln} x_{i_{1}}\right)
$$

The risk index $y_{i j}$ of the negative index is as follows:

$\mathrm{yij}_{\mathrm{ij}}=\left(\operatorname{maxx}_{\mathrm{ij}}-\mathrm{x}_{\mathrm{ij}}\right) /\left(\operatorname{maxx}_{\mathrm{il}}-\operatorname{minx}_{\mathrm{il}}\right)$

Where ${ }^{\mathbf{x}_{\mathrm{ij}}}$ is the value of the ith index of the evaluated object; ${ }^{\text {minx }_{\mathrm{HI}}}$ is the minimum of the risk;
is the maximum of the risk.

\section{The Comprehensive Evaluation of Indexes}

On the basis of determining the quantitative method of the index and index weight, the network public opinion of the tourism crisis event's risk index TRI is represented as:

$$
\operatorname{TRI}=\sum \mathrm{m}_{\mathrm{H}_{1}}=\sum \mathrm{w}_{\mathrm{H}_{1} \mathrm{Y}_{1}}
$$

Where the values of TRI are between $[0,1]$, with the value increases, the risk rises. And when TRI $=1$, it is unsafe. $\mathrm{w}_{\mathrm{ij}}$ is the weight of the ith indicator. The standards of the public opinion risk for 5 grades and their corresponding warning level is as follows in table 1.

\begin{tabular}{|c|c|c|}
\hline Risk index & The warning level & Signal \\
\hline$[0,0.25]$ & The ideal security & green \\
\hline$\left(\begin{array}{ll}0.25, & 0.45)\end{array}\right.$ & Safer & blue \\
\hline$[0.45,0.55]$ & Critical safety & yellow \\
\hline$\left(\begin{array}{ll}0.55, & 0.75)\end{array}\right.$ & Little safe & orange \\
\hline$[0.75,1]$ & Not safe & red \\
\hline
\end{tabular}

Table 1 The hierarchy of risk public opinion

\section{The Case Research}

For an example, In the March to May of 2013 in Hunan, the ticket fee events of the ancient city phoenix's scenic spot, the index system of the network public opinion of tourism crisis event's monitoring and warning has been for a validation. Where the data is from the portal website, Wei Bo, search engines and other channels. From the date of the announcement, it starts recording the daily developments and the changes of public opinion, the public opinion risk index were calculated according to the index system, and the trends of public opinion risk is seen in figure 3. 


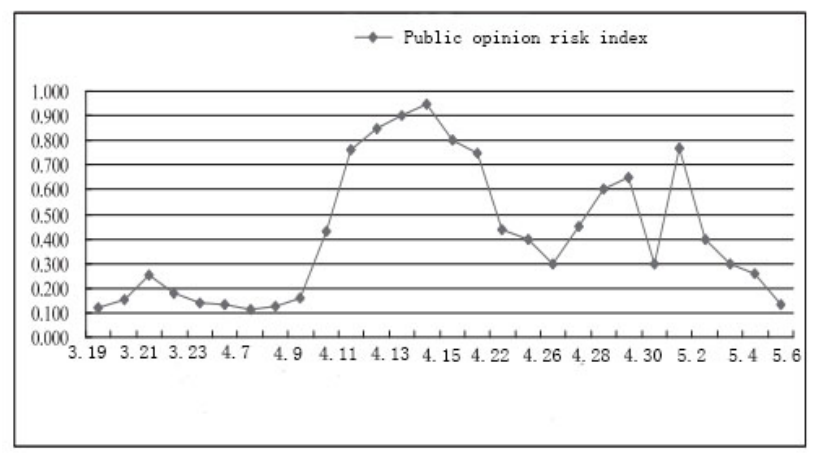

Fig. 3 Trends of public opinion risk

\section{Conclusion}

This study is based on the literature analysis and summarize and the modified Delphi's expert questionnaire and consulting, from the perspective of the form of network public opinion of the tourism crisis events' subject, object and basic ontology, we design the index system of network public opinion monitoring and early warning. After the assignment and quantification for 34 indicators, the index weight of all levels is given by analytic hierarchy process. The importance of these indicators also reflected the tourism crisis events in the new characteristics of network new media environment.

\section{References}

[1] G.X. Tan: Research of Public Emergency Network Public Opinion Monitoring Index System, Vol. 49 (2010) No.3, p.15-19, p.66-70. (In Chinese)

[2] Y. Cheng, C.L. Li: The Threat Assessment Model of Network Public Opinion Based On TOPSIS Method, Vol. 31 (2012) No.3, p.15-19. (In Chinese)

[3] C.S. Gao, C Yue: The Research of Micro-blogging Public Opinion Monitoring Index System , Vol. 30 (2011) No.9, p.66-70. (In Chinese)

[4] Y. W Zhang, J.Y. Qi: The Construction of Unconventional Emergency Network Public Opinion Heat Evaluation Index System, Vol. 29 (2010) No.11, p.71-75. (In Chinese)

[5] Z. T. Du, X. Z. Xie: Using grey prediction and pattern recognition method to build the network public opinion forecast and early warning model, Vol. 57 (2013) No. 15, p. 27-33. (In Chinese). 